\title{
Inadequate Ultrafiltration
}

National Cancer Institute

\section{Source}

National Cancer Institute. Inadequate Ultrafiltration. NCI Thesaurus. Code C62827.

Problem associated with the transfer of fluid between the blood and dialysate through the dialysis membrane due to a pressure gradient (trans-membrane pressure) existing between the blood and dialysate compartments. 